\title{
XXXI. Right ascension, declination, and passage of the Meridian of Ceres; and true apparent Right Ascension of Dr. Maskelyne's 36 stars for every day in the year 1821
}

\section{Rev. J. Grooby}

To cite this article: Rev. J. Grooby (1821) XXXI. Right ascension, declination, and passage of the Meridian of Ceres; and true apparent Right Ascension of Dr. Maskelyne's 36 stars for every day in the year 1821, Philosophical Magazine Series 1, 57:275, 197-199, DOI: $10.1080 / 14786442108652485$

To link to this article: http://dx.doi.org/10.1080/14786442108652485

$$
\text { 曲 Published online: } 27 \text { Jul } 2009 .
$$

Submit your article to this journal ए

$$
\text { Цll Article views: } 2
$$

Q View related articles ¿ 
Geocentric Places of Vesta and Ceres.

exactly one and a half of such water of ammonia. 100 grdins of this water contain $147 \cdot 2$ cubic inches of gas. Hence one grain of water holds condensed in such aqueous ammonia, two cubic inches of the gas ; or one volume of distilled water is united to 505 volumes of gas.

It is a remarkable coincidence, that one volume of water when converted into aqueous muriatic acid, specific gravity 1.200 ; or into aqueous ammonia, specific gravity 0.900 , expands in either case into a volume and a half.

If from 998 we deduct the specific gravity of water of ammonia, expressed in three integers, the remainder, divided by 4 , will give for a quotient the quantity of alkali contained in all such liquid ammonia as is used in medicine, or in chemical researches, viz. between specific gravities 0.936 and 0.980 .

XXXI. Right Ascension, Declination, and Passage of the Meridian of Ceres; and true apparent Right Ascension of Dr. Maskelyne's 36 Stars for every Day in the Year 1821. By the Rev. J. Grooby.

[Continued from p. 133.]

Right Ascension, छัc. of Ceres.

\begin{tabular}{|c|c|c|c|}
\hline 1821. & Rt. Asc. & Declin. & $\begin{array}{l}\text { Passage } \\
\text { of the } \\
\text { Meridian. }\end{array}$ \\
\hline $\begin{array}{r}\text { May } \\
1 \\
7 \\
13 \\
19 \\
25\end{array}$ & $\begin{array}{rr}\text { H. } & \text { M. } \\
16 & 23 \\
16 & 18 \\
16 & 13 \\
16 & 7 \\
15 & 58\end{array}$ & $\begin{array}{cc}14 & 48 \mathrm{~s} . \\
14 & 51 \\
14 & 54 \\
14 & 57 \\
15 & 2\end{array}$ & \begin{tabular}{cc}
\multicolumn{2}{c}{ м. } \\
13 & 49 \\
13 & 15 \\
12 & 56 \\
12 & 23 \\
11 & 51
\end{tabular} \\
\hline $\begin{array}{r}\text { June } 1 \\
7 \\
13 \\
19 \\
25\end{array}$ & $\begin{array}{ll}15 & 52 \\
15 & 46 \\
15 & 42 \\
15 & 38 \\
15 & 35\end{array}$ & $\begin{array}{ll}15 & 11 \\
15 & 21) \\
15 & 30 \\
15 & 42 \\
15 & 56\end{array}$ & $\begin{array}{rr}11 & 14 \\
11 & 40 \\
10 & 6 \\
10 & 42 \\
9 & 19\end{array}$ \\
\hline
\end{tabular}




\section{True apparent Right Ascension of Dr. Maskelyne's 36 Stars.}

\begin{tabular}{|c|c|c|c|c|c|c|c|c|}
\hline 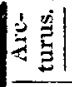 & $\begin{array}{l}-1 \\
4\end{array}$ & 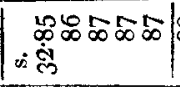 & $\mid$ & கெळळळ & ৪৪৪৪৪ & ৪৪৪৪: & $\infty \infty_{\infty} \infty \infty$ & \\
\hline 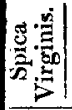 & $\begin{array}{l}\dot{\Delta}: 2 \\
\dot{1}=2\end{array}$ & $\mid \begin{array}{l}\text { T⿱宀八 } \\
\text { मे }\end{array}$ & 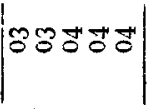 & ठ゙ずずず & |゙ずすの゙ & 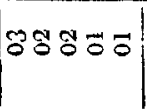 & 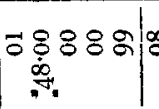 & \% \\
\hline 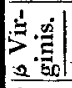 & $\begin{array}{l}\dot{\vec{A}} \vec{\forall} \\
\dot{\mathbf{x}}=\end{array}$ & 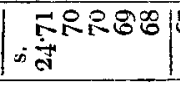 & $\mid 65888$ & $\mid 28080$ & |요용 & | & 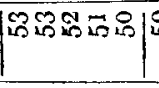 & in \\
\hline$\frac{d}{a}$ & $\begin{array}{l}\dot{8} \\
\dot{1}=\end{array}$ & 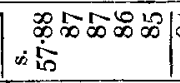 & 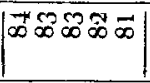 & $\mid$ & 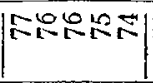 & 프묭 & 1805801 & |B' \\
\hline$=\frac{4}{3}$ & 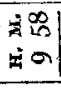 & $\mid \frac{\infty}{\infty} \infty$ & 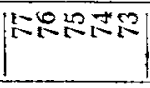 & | & 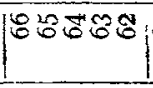 & 689006 & ํำ & in \\
\hline 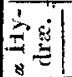 & 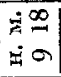 & 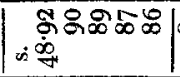 & 1000000 & Rogn & |NR요 & 06280 & 1068.809 & in \\
\hline$\dot{B}$ & 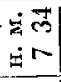 & 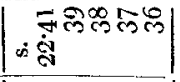 & लिख्युल & 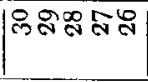 & 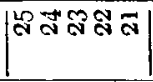 & |웜요 & 1000521 & 19 \\
\hline 总总 & 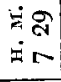 & 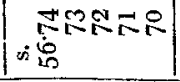 & 80580 & 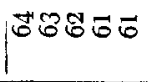 & 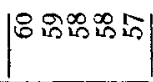 & 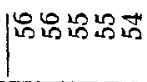 & 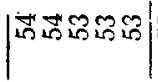 & 58 \\
\hline 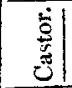 & $\mid \begin{array}{l}n \\
i \\
i\end{array}$ & 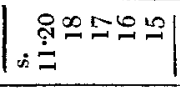 & $\stackrel{2}{2} ニ \cong 8$ & 185820 & | & 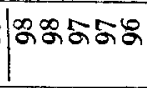 & 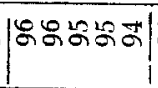 & హ゙ \\
\hline 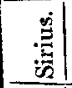 & $\begin{array}{l}\text { मू } \\
\text { in }\end{array}$ & 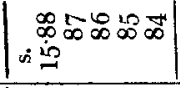 & $\infty \infty \infty \infty$ & RENRK & 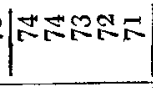 & 은오용 & $|8 x 855|$ & 15 \\
\hline ปั & 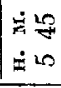 & ๘ & 6888 & 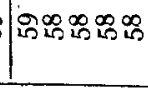 & 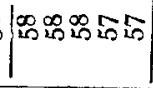 & 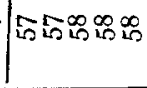 & $\mid \begin{array}{l}0 \\
10\end{array}$ & 8 \\
\hline $2 \cdot \dot{\vec{\Xi}}$ & $\mid \begin{array}{l}\dot{x}= \\
\dot{x}^{20}\end{array}$ & 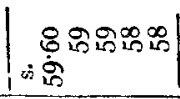 & 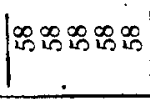 & 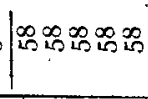 & 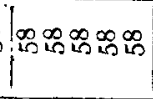 & क & 106800 & $\mathscr{s}$ \\
\hline 离 & 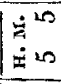 & 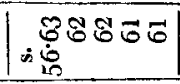 & 68888 & 188888 & 188888 & 1860.8 & 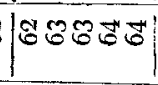 & 18 \\
\hline 采焉 & $\begin{array}{l}\text { मिं } \\
\text { ज्ञ } 10\end{array}$ & 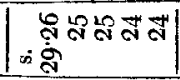 & |ేస్య య & 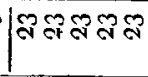 & $\mid \vec{N} \mathbb{゙} ゙$ & 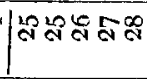 & 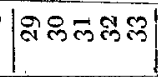 & ले \\
\hline है & 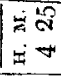 & 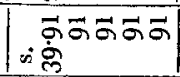 & 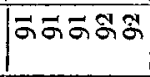 & |รูのゴず & 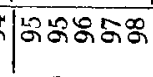 & $1885 \%$ & |3508\% & $\infty$ \\
\hline ن. & 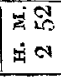 & 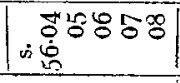 & $890=\mathrm{N}$ & $920 \infty 9$ & สสฺ่ส & क्लेल & 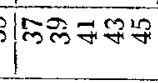 & 18 \\
\hline 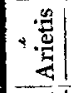 & 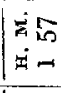 & ஸ્山 & ख़ल खूल & 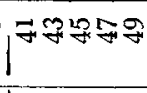 & $\operatorname{lin} 30080$ & $10658=$ & 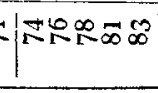 & $\infty$ \\
\hline 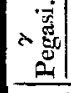 & $\begin{array}{l}\dot{4}+ \\
\text { पi }\end{array}$ & 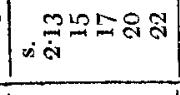 & 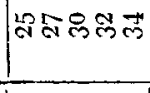 & 궁웜염 & 同 & 1BSRM⿻ & ! $2 \infty_{\infty}^{\circ \infty}$ & D \\
\hline & & 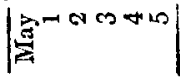 & |or $\infty$ or & 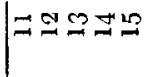 & $10=\infty 90$ & 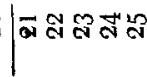 & م. 주 & $\overline{c 5}$ \\
\hline
\end{tabular}


True apparent Right Ascension of Dr. Maskelyne's 36 Stars. 199

\begin{tabular}{|c|c|c|c|c|c|c|}
\hline 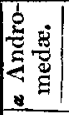 & 在路 & 的 & గீ口卄 & ஜெ こே & 5: & $c i$ \\
\hline 悹 & & $\bar{\infty}$ & 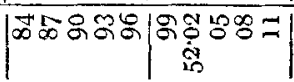 & 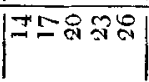 & & F的的的8 \\
\hline | & 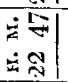 & 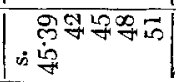 & $6 \mid$ & 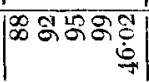 & & \\
\hline 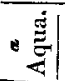 & 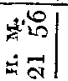 & 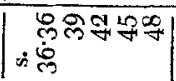 & $\mid$ का in 50808 & & $\mid \begin{array}{c}50 \\
\dot{m}\end{array}$ & $\approx \pi$ \\
\hline 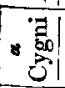 & 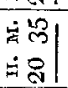 & 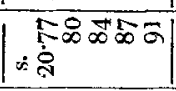 & 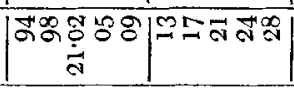 & 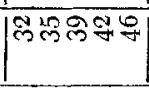 & F & 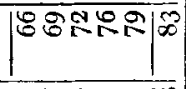 \\
\hline 詈. & 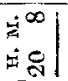 & 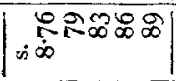 & 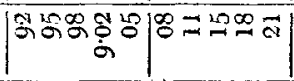 & तై & 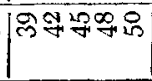 & 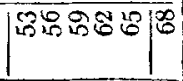 \\
\hline च & 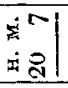 & 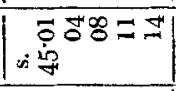 & 다에 & 20 & 年 & 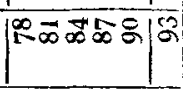 \\
\hline & 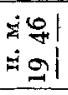 & | & 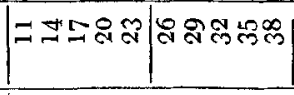 & & 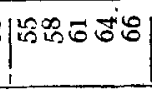 & $\mid 8$ |ะกระ \\
\hline & $\begin{array}{l}+ \text { 이 } \\
\text { जी }\end{array}$ & Lि: & $\infty 20$ & & & F品保 \\
\hline 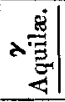 & $\begin{array}{l}40 \\
409 \\
409\end{array}$ & | & 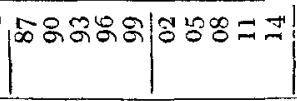 & $\infty_{-\infty}^{\infty}$ & 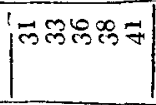 & 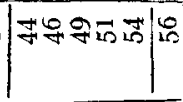 \\
\hline 音 & 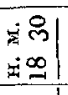 & 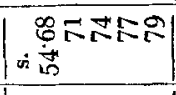 & 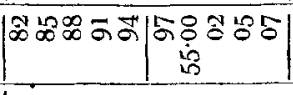 & 58 & & 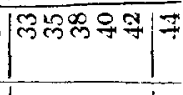 \\
\hline i) & 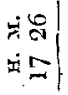 & 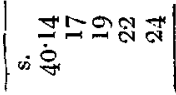 & $1 \infty=9$ & F⿻二丨凵口. & $\mid=8858$ & |ํㅡㅇ유조 \\
\hline 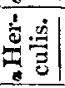 & $\begin{array}{l}50 \\
-10\end{array}$ & 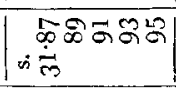 & $\left|\begin{array}{c}580 \\
0 \\
0\end{array}\right|$ & 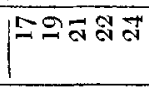 & 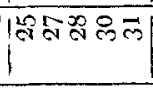 & $\mid$ लिखल्लm|s \\
\hline | & 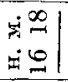 & 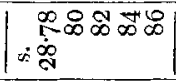 & 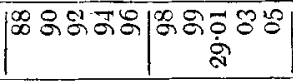 & $599=9$ & 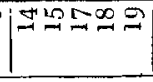 & $\mid 8 \pi \approx \pi$ \\
\hline | & 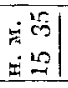 & $\mid \begin{array}{l}\infty \\
\dot{m} \\
\dot{0}\end{array}$ & 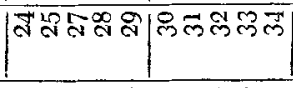 & एक्ष लूळ & & 연 \\
\hline & 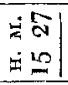 & & 198088160 & 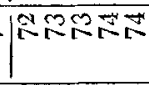 & & 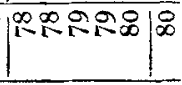 \\
\hline | & \begin{tabular}{l}
-7 \\
\hdashline$=1$
\end{tabular} & & 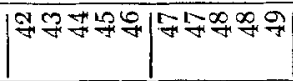 & 웡ํำ的的 & ถู่ง & 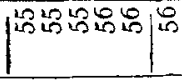 \\
\hline 8 & 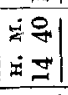 & 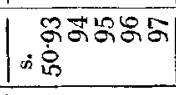 & $\left|\frac{1080}{6}\right|$ & 108855 & 198009 & 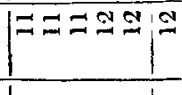 \\
\hline & & | $=-$ - बल & & & & स्रेंक \\
\hline
\end{tabular}

